\title{
Risk Assessment and Gene Flow: Implication for Genetic Resource Conservation (A Review Article)
}

\author{
Temesgen Bedassa Gudeta \\ Department of Biology, Madda Walabu University, P.O. Box: 247, Bale-Robe, Ethiopia.
}

\begin{abstract}
In this review the primary strategies employed in compiling a risk assessment for gene flow from a GM crop to a wild crop relative are outlined. A risk is defined as the likelihood of harm resulting from exposure to the hazard. The spread of new genes and genetic exchange between crops and their wild relatives by introgressive hybridization has been a reality ever since the birth of agriculture. The significance of such gene flow has received little interest, however, until the advent of molecular biotechnology and the emergence of genetically modified (GM) crops. In principle, the scope for the transfer of certain, completely novel transgenes from GM crops into wild relatives means that there is an increased capacity for rapid jumps in the adaptation profile of the recipient. This capacity carries risks for the genetic integrity and evolutionary survival of the wild species, and of the survival of other species with which it coexists. In evaluating such potential problems, one must first define the unwanted outcomes (hazards) as clearly as possible, and then measure the likelihood of their occurrence (exposure). The assessment of risk can be compiled (Risk $=$ hazard $\times$ exposure). Risk is estimated as the product of the probability of exposure, $P(E)$, and the conditional probability of harm given that exposure has occurred, $P(H \mid E)$. That is, $R=P(E) \times P(H \mid E)$. In measuring risks in this way, it is important to consider the management of the crop, the ecology, demography and population genetics of the wild species, the number, nature and location of the transgene(s) and the extent of introgression within the natural setting. Crucially, these assessments should be made against comparable risks posed by non-GM cultivars of the same crop (i.e. baseline data) so that judgements can be made concerning the significance of any change envisaged.
\end{abstract}

Keywords: Conservation, Gene flow, Genetic resources, Hazards, Risks

DOI: $10.7176 /$ ALST/73-02

Publication date: April $30^{\text {th }} 2019$

\section{Introduction}

Modern biotechnology has allowed the movement of genetic material across unrelated species, something impossible with the traditional breeding methods. This intentional transfer of genetic material has in turn brought biotechnology out from the laboratory to the field. Genetically modified (GM) crops are organisms whose genetic material has been artificially modified to change their characteristics in some way or another $[1,2]$. In essence, "genetic modification" or "genetic engineering" techniques enable scientists to find individual genes that control particular characteristics, separate them from the original source, and transfer them directly into the cells of an animal, plant, bacterium, orvirus. Exact probabilities of risk are difficult or impossible to determine for all types of possible harm. Indeed, it is unlikely that all possible harms would be known a priori, particularly with respect to any indirect effects. Hence, it may be necessary - based on current knowledge of population genetics, population dynamics, receiving ecological communities and experience with cultured stocks to classify levels of concern regarding likely genetic impacts posed by cultured stocks into qualitative categories ranging from low to high $[3,4]$. Spontaneous hybridization between crops and wild relatives is neither new nor unusual, and has been reported for almost all food crops over at least part of their cultivated range $[1,5 \& 6]$. It was not until the advent of genetically modified (GM) crops, however, that interest developed in the possible ecological or evolutionary consequences of genetic exchange between a crop and its wild relatives. Over the last decade or so, these concerns have spawned a weighty literature focused on identifying, estimating or measuring the extent of gene movement, and on trying to develop protocols to predict the most probable ecological outcomes.

\section{Contemporary situation of GM technology}

Though modern biotechnology has many potential applications, these new opportunities bring along greater public scrutiny and government regulation. Risk assessment is a common regulatory tool used in the decisionmaking process for a proposed commercial release of a GMO into the environment [3, 4]. Natural hazards have caused severe consequences to the natural, modified and human systems in the past. These consequences seem to increase with time due to both the higher intensity of the natural phenomena and the higher value of elements at risk. The area of land assigned to the commercial production of GM crops has grown steadily since the initial release in 1996, and currently stands at over 80 million hectares globally, an increase of $20 \%$ on that grown in $2003[7,8]$. Over a similar timeframe, advances in the technology have been such that effective transformation systems are now in place for all major and most minor food crops. Nevertheless, exploitation of the technology has been markedly skewed, with just four countries accounting for most of the land area under GM crop 
cultivation (Argentina, Canada, China and USA), four GM crops dominating (maize, cotton, soya and oilseed rape) and two types of trait (herbicide tolerance and Bt-mediated insect resistance) still accounting for almost all commercial constructs [1-4]. It seems unlikely that this simplified profile will continue for long, or even into the foreseeable future, given the increased willingness of additional countries to sanction the commercialization of transgenic material. Furthermore, recent trends towards producing new GM lines that contain multiple inserts and the development of transgenes that confer new traits mean that regulation will need to become increasingly sophisticated to accommodate the new set of potential hazards that such diversification brings [9-11].

\section{Prospective aspect of Genetically Modified crops on environmental risk assessment}

Decision about risks should not be based on the method modification (classical or modern) but on the quality of the final product. What does the GMO contain, is it safe, and not how was the GMO made? Encouragement of new monitoring and detection methods and tools is therefore vital for assessment, control of environmental, and health impacts as well as collection of ecological knowledge of relevance to future releases $[10,12$ \& 13].Environmental risk assessment must consider the unintended consequences of the environmental release of a transgenic plant, particularly as this may impact on existing agricultural practices and the agro-ecosystem. A non target organism is any plant, animal or microorganism that is unintentionally impacted by the novel, or transgenic, plant. There is currently no globally accepted procedure for the handling of GM cultivars or for their regulation, Figure 1. However, there is broad consensus on the desire to assess each new submission separately on a 'case-by-case' basis [14]. In most countries that permit the cultivation of GM crops, permission for commercialization of a GM line invariably requires provision of extensive data sets relating to the crop, the transgene and the likelihood of gene flow to wild relatives. It is open to question whether such an exhaustive approach will remain tenable indefinitely. Moreover, as the number of crop-GM cultivar-transgene-transgene mixture location combinations grows and as the number of new submissions also increases, so will the pressure on the various regulatory systems to become more streamlined and to focus increasingly on outcomes that are most undesirable or most likely, or a combination. Under these circumstances, the pressure to adopt more generic approaches to risk assessment may become irresistible $(15,16]$.



Figure 1: Framework of risk management

\section{Gene flow from non-GM crops}

According to Ruchir Raman $[2,7]$ there are certain aspects of the transgenes that make GM crops a special case when considering the consequences of gene flow (e.g. the fact that the transgene often originates from very distantly related organisms or can even be synthetic), there are some environmental issues that apply equally to gene flow from conventional (i.e. non-GM) crops and wild relatives. For instance, extensive introgression from a conventional crop could theoretically compromise the genetic integrity of a rare wild relative [12, 17]. It is also plausible that gene transfer from a conventional crop could provide a weedy relative with a new source of herbicide tolerance or insect resistance that had been introduced into the crop from another species. Indeed, the transfer of any gene from a crop that has the capacity to enhance fitness of a wild relative in its existing habitat or else enable it to occupy a new habitat has the potential to cause unwanted ecological change [18]. There are subtle differences in the way that assessment of risk from conventional and GM crops should be handled. For example, in GM cultivars, attention focuses on the transgenes, where extensive information is generally available 
on gene function and (sometimes) on gene position [19]. In conventional cultivars (especially those in which exotic germplasm have featured in their pedigrees), the identity of the gene or genes responsible for the trait of concern is usually unknown. Indeed, even the traits most likely to cause problems after introgression into a relative may themselves be undefined. In spite of such differences, however, the principles underlying the process of predicting the ecological outcome of gene flow remains constant and is outlined below.

\section{Succinct Concept of risk assessment}

The term risk of an element is defined as "the sum of expected losses and damage of any kind due to a particular natural phenomenon, as function of the natural hazard and the vulnerability of the element at risk."As stated by [4, 18 \& 20]. In analogy to the average hazard, the average risk, R(D), can be written mathematically:

$$
R(D)=\int_{0}^{\infty} x \cdot V(x) \cdot f_{D}(x) \mathrm{d} x .
$$

Inpractical terms, risk is the real threat to an element (or a system) given its vulnerability towards the phenomenon [4]. There are three terms that must be considered when performing any biological risk assessment: Hazard, Exposure and Risk. The use of these terms has a strict meaning in a risk assessment context and they must not be used interchangeably. Hazard refers to the adverse effect or unwanted ecological outcome. Ideally, this should be specified as explicitly as possible if the risk assessment is to have value in decision-making. Exposure refers to the extent of contact with the agent or process that may cause the unwanted event (hazard) to occur. In toxicology, this might refer to the exposure of a subject to a chemical agent, but in the context of gene flow it generally refers to several processes relating to gene transfer and their knock-on effects. To paraphrase, exposure is a measure of how likely it is that the unwanted event will occur. Risk represents a consideration of both the magnitude of the unwanted event (i.e. the hazard) and the likelihood that it will occur (exposure). Thus, Risk is a function of both Hazard and Exposure, and can be represented as follows: Risk $=f$ (Hazard, Exposure) or sometimes: Risk $=$ Hazard $\times$ Exposure When performing a risk assessment of gene flow between crop and wild relative, we need to define all plausible Hazard(s) and to quantify the Exposure for each of these Hazards. The first element of this task is the definition of hazards [3, $4 \& 17]$. A globally improved risk mitigation strategy and communication with growers, therefore ensuring greater acceptance.

\section{Crops Based concept of hazards versus gene flow}

Gene flow is the collective term for mechanisms resulting in gene movement between populations of the same or different species or subspecies, and it is the evolutionary force that genetically coheres populations. There are generally several possible unwanted ecological outcomes that require assessment from any particular GM cultivar whenever a cross-compatible wild relative is present [21, 22]. For instance, passage of a transgene conferring insect resistance might reasonably be expected to increase the chances that a cross-compatible relative may become more abundant (i.e. from the release from herbivore pressure). It is equally possible that the presence of resistance against one group of insects (e.g. Lepidopteran species) may change the balance of herbivores, predators and parasites within the community. The number of possible outcomes when one considers all exotic genes introduced into a non-GM crop is far larger and more difficult to specify. Nevertheless, it is possible to classify all hazards into two broad categories: Direct and Indirect. Direct hazards relate to the recipient itself and could include increased (or decreased) population size of the wild relative within its existing habitat, its invasion of a new habitat after acquisition of the crop gene(s) or a genetic sweep causing reduced allelic diversity in genes located around the transferred crop gene(s). Indirect hazards relate to the impact introgressed crop wild relatives (CWRs) have on other plants or animals that interact with the recipient species $[2,16]$. These could include decline in sympatric plant species (through interspecific competition), or changes in the abundance of pollinators, herbivores or predators. It is therefore important to note that the species affected in a negative way (known as the assessment endpoint species) may not be the CWR itself. Thus, the first element of a risk assessment relies on assembling a set of feasible specifically defined hazards that have ecological, social or evolutionary importance to the relative itself and others with which it associates. These hazards should then be ranked and priority given to those hazards deemed to be both likely and important.

\section{Assessment of exposure}

Routes of exposure need to be identified. Exposed organisms are determined through suspected causal chains of impact. Based on this information, protocols and methodologies for appropriate testing can then be developed $[12,17]$. The measurement of exposure to a chemical agent is a relatively simple task, ecological exposure to the effects of gene flow from a crop to a CWR is far more problematic to estimate. This is because the route to ecological harm (Hazard realization) is reliant upon a pathway or matrix of intermediate events. For instance, Figure 2 illustrates a hypothetical pathway of linked events through which movement of an insect resistance transgene from a GM crop could lead to the local extinction of a specialist parasitoid. In reality, of course, there 
are usually several possible hazards that could result from gene flow from a GM (or conventional) crop to a CWR. If a risk assessment is to account for all of these (or at least all deemed important or relevant), then the exposure pathways of several hazards coalesce to form a matrix in which the early stages are shared, but later elements are gene or hazard specific. Estimates of the shared early stages apply to all (or at least most) hazards and so have generic value for most risk assessments. These elements of exposure can therefore be evaluated before prioritization of the hazards identified since they will invariably be important as long as there is one hazard considered to be significant, Figure 2. In contrast, the later elements may relate only to one particular hazard or gene and so may not be evaluated unless the hazard is regarded as a high priority [16, 22].



Figure 2: Sequence of events by which gene flow of a transgene conferring insect resistance could lead to local extinction of a specialist parasitoid (Hypothetical exposure pathway).

\section{Risk assessment course of action}

Risk assessment also focused on the change brought about by genetic engineering allows for detailed consideration of the potential consequences of the change relative to the way the GM plant is intended to be used and the environments in which it may be found. Risk assessment also focused on the change brought about by genetic engineering allows for detailed consideration of the potential consequences of the change relative to the way the GM plant is intended to be used and the environments in which it may be found [2]. The process of assessing risk involves the consideration of all contributing elements in order to enable the decision-making process. In the case of GM cultivars, this information may be used to decide whether to grant permission for commercial cultivation or else to recommend measures to minimize risk (risk management) [4, 11]There are essentially three elements that need to be combined to perform a risk assessment and it is preferable to assess these in the following order:

1. Specify and rank hazards.

2. Quantify generic aspects of exposure.

3. Evaluate transgene or cultivar-specific exposure aspects.

1. Specify and rank hazards

There are four tasks to be completed before hazards relating to a particular crop-location combination can be ranked: identify cross-compatible relatives; rank relatives crudely on gross exposure (sympatry, ease of hybridization); specify direct hazards (relating to a CWR); and specify indirect hazards (relating to sympatric organisms). This process can be usefully illustrated using wheat in the USA as a case study.

Specify direct and indirect hazards (third and fourth tasks): To a large extent, this would depend on the transgene or crop trait being considered. An example of a direct hazard (relating to the CWR) would be that a transgene conferring herbicide tolerance would allow the CWR to escape from agronomic control and so become a more pernicious weed. An example of indirect hazard (relating to another, non-CWR species) would be that 
rapid assimilation of tolerance to a particular herbicide targeted by the transgene will lead to a change in herbicide use that in turn leads to a decline in a non-target plant species or animal species (e.g. through loss of food plant) [11]. For any crop wild relative combination, there will be many possible but fewer probable hazards that might arise as a result of gene flow from the crop. Given that comprehensive assessment of exposure is both slow and expensive, there is a pressing need to rank or prioritize those hazards that have been identified. The rankings generated will inevitably involve an element of subjectivity and will depend heavily on the relative importance that assessors or regulators place on factors such as the scarcity of the end point species (e.g. is the affected species IUCN red-listed?), its cultural importance (e.g. bald eagle in the USA has high social importance), its economic importance (e.g. is it a commercially important weed?), or its agronomic or medicinal importance (e.g. many CWRs have agronomic value in their own right or have value as a source of resistance genes). Interestingly, emphases are likely to change between countries and may vary within a country over time. For this reason, the set of criteria used to compile a ranking and therefore the rankings themselves will also vary [19]. In this context, the resultant risk assessment will reflect the wishes of the regulator at the time and place when the assessment is performed. Nevertheless, risk assessments may also be performed in which priorities are fixed and specific (e.g. risk assessment for the protection of the genetic diversity of CWRs or for the conservation of endangered species).

\section{Quantify generic aspects of exposure}

Having selected the CWR(s) and hazards of greatest concern for a particular region, the next stage is to assess the early (i.e. generic) elements of exposure. There are three generic exposure elements that can be usefully characterized for a particular crop-relative-geographic region combination: initial hybrid formation; introgressive hybridization; and secondary gene spread. It is helpful to assess each in the order in which they occur, since it can be reasonably argued that there is no point in assessing the likelihood of introgression if no hybrids form, or to quantify secondary spread if the hybrids are sterile and unable to produce stable introgressants $[12,17 \& 18]$. In Initial hybrid formation useful information on the frequency of hybrid formation can sometimes be obtained directly from field trials, it is important to remember external factors that can influence hybrid frequency in the natural or agricultural setting. It is certainly important to define the context of contact between crop and its wild relative. Weedy wild relatives will usually come into frequent and intimate contact with the crop and so will usually be more likely to form large numbers of hybrids than relatives occupying wild habitats. Among the latter, it should be noted that some habitats (woodlands, hedgerows, riverbanks and even sea cliffs) are often adjacent to arable land whereas others (alpine, salt marshes, heaths, desert, etc.) are more likely to have significant physical isolation from the crop. Cognisance should also be taken of the regional distribution of the crop and the CWR. There are several approaches that can be employed to access relevant information on distribution, including literature and direct surveys, herbarium screens and even remote sensing [21, 22].

Having established the range and context of contact between crop and the CWR, it is desirable to assess the rate of hybridization under conditions that reflect those encountered in the natural environment. For this, it is vital to consider the relative sizes of the donor fields and recipient populations, and of crop rotation patterns and common agronomic practices (particularly for weeds), since these can profoundly influence hybrid frequency. Once appropriate settings have been established to describe field conditions, attention then turns towards estimating gene flow under these circumstances. Perhaps the simplest empirical approach to estimating gene flow is to collect seed of the recipient CWR and screen for the abundance of hybrid seeds. This is relatively easy to perform provided that there is an effective means of screening large numbers of progeny for hybrids, such as morphological differences or flow cytometry [23]. Confirmation of hybrid identity generally requires application of molecular markers and/or tests for the presence of the transgene (e.g. ELISA).

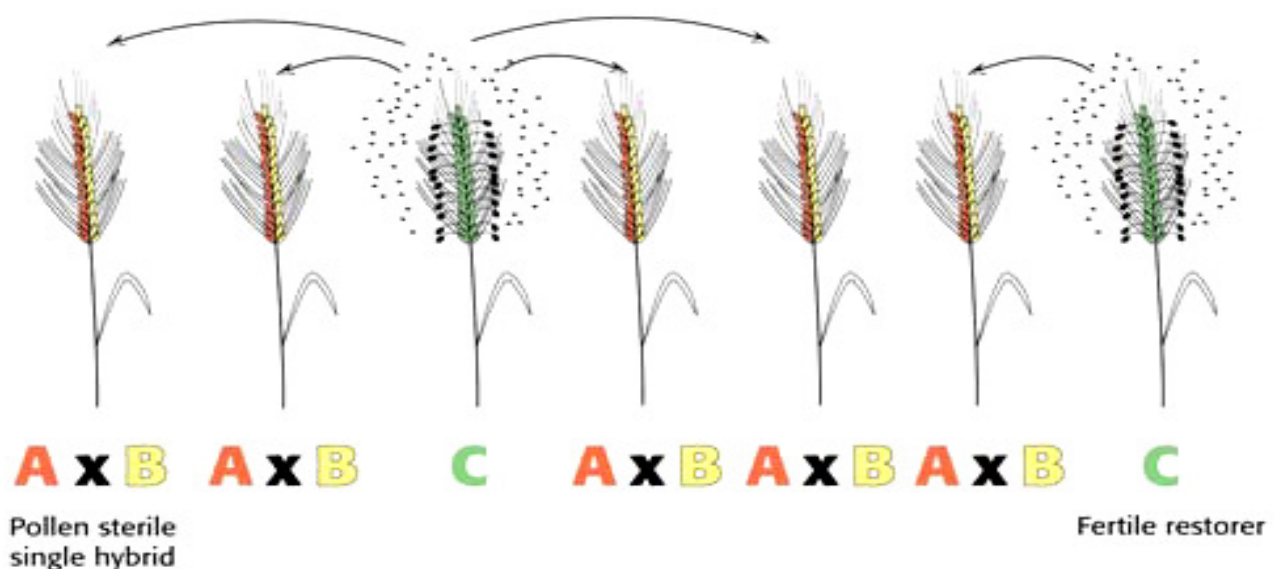

Figure 3: Gene flow, pollen dispersal and seed dispersal models [2] 
Nevertheless, critical limitations lie in the basic assumptions that the dormancy of the hybrid seed is the same as that of the wild relative and that hybrids and wild relative seedlings are equally able to establish themselves in the natural habitat. Another confounding factor relates to the rotation pattern of the crop; this means that hybrids will appear almost exclusively in those years when crop and the CWR are sympatric. As a consequence, hybrid numbers could tend to show cycles of abundance that match the rotation cycle. However, any differences in the dormancy characteristics of the hybrid seeds will dampen the amplitude and distinctness of such cyclical changes in hybrid plant frequency. Modelling can accommodate these factors, although a more direct (albeit more difficult) approach is to record the abundance of hybrid plants among flowering populations of the CWR in sites of previous sympatry. In either case, modelling will still be required to apply any results to a landscape or national scale $[13,21]$. In the absence of direct measures of gene flow, pollen dispersal and seed dispersal models can be used as crude guides to the patterns, range and approximate scale of hybrid formation, Figure 3:. When assembling large-scale models of any kind, it is vitally important that one gains a realistic picture of the rotation patterns adopted by farmers in the region of interest. This can be obtained by reference to farmer's records, or by remote sensing when the crop has a distinctive multispectral mark.

Introgressive hybridization: There have been several reports providing evidence of introgression between crops and wild relatives [6,7] few have adopted a mapped-based approach to characterize the nature of the introgressed segments. These works have innate value in their own right, but do not provide vital information on the likelihood of a particular locus being transferred. This is particularly important if linkage drag around deleterious crop genes and drive around advantageous ones mean that some regions of the genome are very much more likely to be stably introgressed than others. This is also true where some regions of the wild relative genome show greater or reduced homology to the crop genome than others, and so are more or less likely to pair at meiosis. In the future, therefore, it is likely that various approaches will be adopted to identify genomic regions with a high likelihood of introgression. Such information might be gained by reference to existing mapping and breeding data (attempts to introduce desirable genes from wild relatives into the crop) or might instead relyon the application of linkage disequilibrium-based strategies to characterize historic gene flow ( 7,18 \& 25]

\section{Evaluate transgene -or cultivar-specific exposure aspects}

Gene flow is the route along which transgenes can spread genetically into populations of related species and geographically into other regions including protected areas of sensitive ecological value. Gene flow is considered a risk because of the great uncertainties that are associated with the consequences this might induce in the recipient ecosystems. In many cases, it would be a daunting task to provide quantitative predictions because of the complexity of interactions between organisms at a community level. However, the purpose of risk assessment is not to provide realistic and predictive ecological models; rather, it is a tool to assist the decisionmaking process [12].Thus, it is important only to establish whether or not something is very unlikely to happen. Freedom from the need to provide truly predictive estimates of exposure has allowed a tiered approach to be developed in which the hazard is first mimicked under 'worst-case scenario' conditions (tier 1 experiments). If the hazard is not realized under these situations, the exposure is deemed to be negligible. However, if the hazard is realized, then more complex (tier 2 and tier 3 ) experiments are performed in which the conditions become increasingly more similar to that encountered in the wild. The value of the tiered approach can perhaps be illustrated by the possible hazard that Lepidopteran-specific Bt resistance in maize pollen could cause a crash in North American populations of the Monarch butterfly. Losey and colleagues [26] used simple laboratory feeding experiments (tier 1) to indicate that the presence of pollen from GM maize could negatively influence survival of Monarch butterfly larvae when dusted on their normal food plant. The authors inferred that the presence of GM Bt maize could therefore constitute a threat to the long-term survival of the Monarch in North America [27]. Whilst this work was subject to criticism the identification of this potential hazard led to an excellent series of tier 2 and 3 'exposure' papers that evaluated the likelihood that the hazard would occur [28, 29]. These efforts finally led to the risk being assessed as negligible that GM Bt maize would cause serious decline in Monarch numbers in the USA [30]. The tiered testing approach has been used widely in the testing of pesticides. A moreor-less standard procedure is now used and accepted within the European Union [31]. Several authors have called for a tiered approach to be routinely adopted for the risk assessment of GM crops [15, 32]. The same approach could equally be applied to hazards arising from gene flow from non-GM crops. Successful transgene flow will affect simultaneously both recipient plants and their associated organisms. Protocols need to be developed for establishing for hazards identified as high priority and where hybridization, introgression and spread are considered inevitable it may become important to assess the later elements of exposure.

\section{Conclusion}

The probability and consequences of gene flow to wild relatives is typically considered in the environmental risk assessment of genetically engineered crops. The process of assessing ecological risks of gene flow between crops and their wild relatives is complex and requires a structured, sequential approach. Initial work should focus 
on the identification and ranking of CWRs in a region and of the hazards that could conceivably result from the stable transfer of a gene or genes into these species. Having established the identity of high priority hazards, emphasis should turn to measuring the likelihood of occurrence (exposure). Ecological exposure differs from exposure to toxic chemicals in that it comprises of a series of events that must occur in a particular sequence in order that the hazard is realized. In the risk assessment of a particular crop-gene-wild relative-location combination, there will generally be several potential hazards worthy of study. With respect to gene flow, all will share three common elements of exposure, namely initial hybridization, introgression, and gene spread between populations. These elements therefore have generic value, and should be evaluated for all wild relatives deemed to be important in an area. The later elements of exposure are specific to one or a few hazards and are more problematic to quantify. However, for risk assessment purposes, a tiered approach provides a useful means of evaluating whether a particular scenario is sufficiently unlikely to justify its being discarded on the basis of low exposure. If not, regulators must make decisions on whether to refuse permission for release or to impose risk management restrictions. Monitoring and detection methods are vital for risk assessment and management to control the negative environmental and health impacts. The international biosafety regulatory frameworks are sufficiently stringent in order to protect against genuine ascertainable risks, as well as the ability of decision makers to discern the appropriateness of data necessary to adequately conduct a risk assessment, which all have considerable consequences. Consideration of social, economic, and ethical issues needs to be taken care of. Application of the precautionary approach provides avenues for future development and use of genetic engineering.

\section{Conflicts of Interest}

The authors declare that there is no conflict of interest.

\section{Acknowledgments}

Although this review article is my own work, it would not have been possible without certain people. I would like to thank the editor and the reviewers from staff members of Biology department of Madda Walabu University for their helpful comments and remarks.

\section{References}

[1]. Tzotzos G. T., Head G. P., and HulR. 1, 2009 ."Principles of risk assessment," Genetically Modified Plants, vol. 2009, pp. 33-63.

[2]. Ruchir Raman (2018). The impact of Genetically Modified (GM) crops in modern agriculture: A review, GM Crops \& Food, 8:4, 195-208, DOI: 10.1080/21645698.2017.1413522

[3]. FAO, 2015. Animal Genetic Resources for food and Agriculture. The Second Report on the State of the World's Issn 2412-5474

[4]. Tsakiris G., 2014. Flood risk assessment: concepts, modelling, applications. Nat. Hazards Earth Syst. Sci., 14,1361 .

[5]. Ellstrand, N.C., Prentice, H.C. \& Hancock, J.F. 1999. Gene flow and introgression from domesticated plants into their wild relatives. Annual Review of Ecology and Systematics, 30: 539-563.

[6]. Stankowski S, Streisfeld MA. 2015. Introgressive hybridization facilitates adaptive divergence in a recent radiation ofmonkeyflowers. Proc. R. Soc. B 282: 20151666. http://dx.doi.org/10.1098/rspb.2015.1666

[7]. Nosil P. 2008. Speciation with gene flow could be common. Mol. Ecol. 17, 2103-2106.

[8]. James, C. 2004. Preview: Global Status of Commercialized Biotech/GM Crops: 2004. International Service for the Acquisition of Agribiotech Applications (ISAAA), Ithaca, NY, USA. ISAAA Briefs, No. 32. $43 \mathrm{p}$.

[9]. Alberghina L., L. Frontali, and P. Sensi, 1994. Proceedings of the 6th European Congress on Biotechnology, Elsevier Science Publishers, Amsterdam, The Netherlands, 1994.

[10]. Hill R. A., 2005 "Conceptualizing risk assessment methodology for genetically modified organisms," Environmental Biosafety Research, vol. 4, no. 2, pp. 67-70.

[11]. Kuiper H. A. and Davies H. V., 2010. "The safe foods risk analysis framework suitable for GMOs? A case study," Food Control, vol. 21, no. 12, pp. 1662-1676.

[12]. Raybould, A.F. \& Wilkinson, M.J. 2005. Assessing the environmental risks of gene flow from GM crops to wild relatives. pp. 169-185, in: G.M. Poppy and M.J. Wilkinson (editors). Gene Flow from GM Plants. Blackwell Publishing, Oxford, UK.

[13]. Dhan Prakash, Sonika Verma Ranjana Bhatia and B. N. Tiwary, 2011. Risks and Precautions of GeneticallyModified Organisms. International Scholarly Research Network ISRN Ecology Volume 2011.

[14]. Manjunath J Shetty, Chandan K, HC Krishna and Aparna GS, 2018. Genetically modified crops: An overview. Journal of Pharmacognosy and Phytochemistry; 7(1): 2405-2410

[15]. Wilkinson, M.J., Sweet, J. \& Poppy, G.M. 2003a. Risk assessment of GM plants: avoiding gridlock? 
Trends in Plant Science, 8: 208-212.

[16]. Centre for Food Safety. Proposed Oregon legislation would allow communities to protect Farmers' traditional crops from contamination by genetically engineered crops. 2016. Available at: https://www. centerforfoodsafety.org/press-releases/4211/pro protect-farmers-traditional-crops-from-contamina posed-oregon-legislation-would-allow-communitiesto17March 2019].

[17]. Garcia-Alonso M, Raybould A., 2014. Protection goals in environmental risk assessment: a practical approach. Transgenic Res 23:945-956

[18]. Smyth S., 2017. Genetically modified crops, regulatory delays, and international trade. Food and Energy Security, 6(2):78-86.

[19]. Genetic Literacy Project. Where are GMOs grown and banned?. 2018. Available at: https://gmo.geneti cliteracyproject.org/FAQ/where-are-gmos-grownand- banned/ [Accessed 14 January, 2019].

[20]. Nicolosi, V., Cancelliere, A., and Rossi, G., 2007. Drought risk analysis in water supply systems using genetic algorithms and Monte Carlo simulation, in: Proc. of EWRA Symposium "Water Resources Management: New Approaches and Technologies", Chania, Crete-Greece, 14-16 June 2007, 33-40.

[21]. Wilkinson, M.J., Elliott, L.J., Allainguillaume, J., Shaw, M.W., Norris, C., Welters, R., Alexander, M., Sweet, J. \& Mason, D.C. 2003b. Hybridization between Brassica napus and B. rapa on a national scale in the United Kingdom. Science, 302: 457-459.

[22]. Elliott, L., Mason, D., Wilkinson, M.J., Allainguillaume, J., Norris, C., Alexander, M. \& Welters, R. 2004. Using satellite image processing for large-scale study of gene flow from genetically modified rapeseed. Journal of Applied Ecology, 41: 1174-1184.

[23]. Sears, M.K., Hellmich, R.L., Stanley-Horn, D.E., Oberhauser, K.S., Pleasants, J.M., Mattila, H.R., Siegfried, B.D. \& Dively, G. 2001. Impact of Bt corn pollen on monarch butterfly populations: A risk assessment. Proceedings of the National Academy of Scence of the USA, 98: 11937-11942.

[24]. Andersen, N.S., Siegismund, H.R., Meyer, V. \& Jorgensen, R.B. 2005. Low level of gene flow from cultivated beets (Beta vulgaris L. ssp. vulgaris) into Danish populations of sea beet (Beta vulgaris L. ssp. maritima (L) Arcangeli. Molecular Ecology, 14: 1391-1405.

[25]. Richard G. Harrison and Erica L. Larson, 2014. Hybridization, Introgression, and the Nature of Species Boundaries. Journal of Heredity 2014:105(Special Issue):795-809 doi:10.1093/jhered/esu033

[26]. Losey, J.E., Rayor, L.S. \& Carter, M.E. 1999. Transgenic pollen harms monarch larvae. Nature,399: 214.

[27]. Hodgson, J. 1999. Monarch Bt-corn paper questioned. Nature Biotechnology, 17: 627.

[28]. Stanley-Horn, D.E., Dively, G., Hellmich, R.L., Mattila, H.R., Sears, M.K., Rose, R., Jesse, L.C.H., Losey, J.E., Obrycki, J.J. \& Lewis, L. 2001. Assessing the impact of Cryl Ab-expressing corn pollen on monarch butterfly larvae in field studies. Proceedings of the National Academy of Scence of the USA, 98: 1193111936.

[29]. Oberhauser, K.S., Prysby, M.D., Mattila, H.R., Stanley-Horn, D.E., Sears, M.K., Dively, G., Olson, E., Pleasants, J.M., Lam, W.K.F. \& Hellmich, R.L. 2001. Temporal and spatial overlap between monarch larvae and corn pollen. Proceedings of the National Academy of Scence of the USA, 98: 11913-11918.

[30]. Gatehouse, A.M.R., Ferry, N. \& Raemaekers, R.J.M. 2002. The case of the Monarch Butterfly: a verdict is returned. Trends in Genetics, 18: 249-251.

[31]. EPPO [European and Mediterranean Plant Protection Organization]. 2003. Environmental risk assessment scheme for plant protection products. Chapter 7: Aquatic organisms. EPPO Bulletin, 33: 183-194.

[32]. Poppy, G.M. 2000. GM crops: environmental risks and non-target effects. Trends in Plant Science, 5: 4-6. 